\title{
Relationship-centered communication training for pediatric hematology/oncology providers to improve patient experience
}

\author{
${\text { Christine } \text { Yost }^{1} \text {, Rosa Banuelos }}^{2}$, Joan Shook ${ }^{1}$, and Mary Shapiro ${ }^{3}$ \\ ${ }^{1}$ Baylor College of Medicine \\ ${ }^{2}$ Texas Children's Hospital \\ ${ }^{3}$ Texas Children's Cancer Center and Hematology Centers
}

October 13, 2020

\begin{abstract}
Background Pediatric hematology/oncology providers frequently have challenging discussions with patients/families, yet receive little formal training in communication. Our institution aimed to meet this need with a workshop in relationship-centered communication (RCC), which focuses on empathy, reflective listening, and patient/family understanding. This study evaluates the efficacy of this intervention to improve patient/family experience in healthcare encounters. Procedure Our team at Texas Children's Hospital (TCH), a quaternary care pediatric and women's hospital, developed a 5.5-hour RCC skills workshop in collaboration with the Academy of Communication in Healthcare (ACH). ACH-trained TCH providers led participants in 3 pediatric-specific skill sets that incorporated didactics, role-playing skills practice, and reflective feedback. Patient experience was measured using Press Ganey email surveys (PGS) that were collected for a minimum of 12 months pre-course and 3 months post-course. Results Forty-nine pediatric hematology/oncology providers voluntarily participated in the RCC skills workshop over a 3-year period. The majority of participants were physicians (77.6\%), female (85.7\%), and in the first 10 years of practice $(61.2 \%)$. Of the 15 participants who had both pre- and post-course PGS available, scores significantly improved in all areas of the care provider section $(\mathrm{P}<0.05)$. Conclusions RCC skills training improved pediatric hematology/oncology patient/family experience.
\end{abstract}

Title: Relationship-centered communication training for pediatric hematology/oncology providers to improve patient experience

Authors : Christine Yost, MD, Rosa C. Banuelos, PhD, Joan E. Shook, MD, MBA, and Mary Carroll Shapiro, MD

\section{Author affiliations :}

Christine Yost, MD, Department of Pediatrics, Baylor College of Medicine

Rosa C. Banuelos, PhD, Texas Children's Hospital Quality Outcomes and Analytics

Joan E. Shook, MD, MBA, Department of Pediatrics, Baylor College of Medicine

Mary Carroll Shapiro, MD, Texas Children's Cancer and Hematology Centers, Department of Pediatrics, Baylor College of Medicine

\section{Corresponding author :}

Mary Carroll Shapiro, MD

6701 Fannin Street, Mark Wallace Tower, Suite D.1510.23, Houston, TX 77030 
Phone 832-824-4751

Fax 832-227-1096

mmcarrol@texaschildrens.org

Abstract : 194 words

Main Text : 1566 words (excludes Title Page, Abstract, References, Tables, Figures, and Legends)

Tables : 2

Figures : 1

Supporting Information files : 0

Running title: RCC training and patient experience in PHO

Keywords: communication training, relationship-centered communication, patient experience, hematology/oncology, general

\section{Abbreviations:}

\begin{tabular}{ll}
\hline ACH & Academy of Communication in Healthcare \\
\hline NCI & National Cancer Institute \\
PGS & Press Ganey surveys \\
RCC & Relationship-centered communication \\
TCH & Texas Children's Hospital \\
\hline
\end{tabular}

\section{Note.}

"Relationship-centered communication training to improve patient experience and provider burnout." The American Society of Pediatric Hematology and Oncology 33 ${ }^{\text {rd }}$ Annual Meeting Poster Presentation; 05.08.20 (cancelled due to SARS-COV-2 pandemic).

"Relationship-centered communication training to improve patient experience and provider burnout." Pediatric Academic Societies Meeting Poster Presentation; 05.02.20 (cancelled due to SARS-COV-2 pandemic).

\section{Abstract \\ Background}

Pediatric hematology/oncology providers frequently have challenging discussions with patients/families, yet receive little formal training in communication. Our institution aimed to meet this need with a workshop in relationship-centered communication (RCC), which focuses on empathy, reflective listening, and patient/family understanding. This study evaluates the efficacy of this intervention to improve patient/family experience in healthcare encounters.

\section{Procedure}

Our team at Texas Children's Hospital (TCH), a quaternary care pediatric and women's hospital, developed a 5.5-hour RCC skills workshop in collaboration with the Academy of Communication in Healthcare (ACH). ACH-trained TCH providers led participants in 3 pediatric-specific skill sets that incorporated didactics, role-playing skills practice, and reflective feedback. Patient experience was measured using Press Ganey email surveys (PGS) that were collected for a minimum of 12 months pre-course and 3 months post-course.

\section{Results}

Forty-nine pediatric hematology/oncology providers voluntarily participated in the RCC skills workshop over a 3-year period. The majority of participants were physicians (77.6\%), female (85.7\%), and in the first 10 
years of practice $(61.2 \%)$. Of the 15 participants who had both pre- and post-course PGS available, scores significantly improved in all areas of the care provider section $(P<0.05)$.

\section{Conclusions}

RCC skills training improved pediatric hematology/oncology patient/family experience.

\section{Introduction}

Relationship-centered care is defined as an approach that prioritizes both biomedical and psychosocial aspects of the therapeutic relationship between a provider and patient/family. The key dimensions of relationshipcentered care are provider self-awareness/self-growth, the patient's/family's experience of health and illness, and the development and maintenance of relationships through effective relationship-centered communication (RCC). ${ }^{1}$ RCC focuses on empathy, reflective listening, patient/family perspectives, and patient/family understanding of information. ${ }^{1,2}$ A provider's communication approach is a powerful and invaluable skill that can affect both patient/family satisfaction ${ }^{3}$ and patient healthcare outcomes. ${ }^{4}$ Specific to pediatric medicine, effective communication must take place within the patient-parent-provider triad and relies on a foundation of informativeness, interpersonal sensitivity, and partnership building. ${ }^{5,6}$ Furthermore, within pediatric hematology/oncology, the provider's communication approach has the ability to significantly impact complex information sharing and decision-making between the patient/family and provider. $^{7-12}$

In 2007, the National Cancer Institute (NCI) identified 6 core functions of communication in the care of adult patients with cancer:fostering healing relationships, exchanging information, responding to emotions, managing uncertainty, making decisions, andenabling patient/family self-management.$^{13}$ These communication functions have also been validated in pediatrics, with the discovery of additional functions of providing validation and supporting hope that have explicit importance within pediatrics. ${ }^{14}$ There has been some descriptive research regarding communication within pediatric oncology, but there is a need for interventional studies regarding the NCI core communication functions within pediatric hematology/oncology. ${ }^{8}$ In this study, we aim to evaluate the impact of an RCC skills training intervention for pediatric hematology/oncology providers on patient/family experience. Our group has previously demonstrated the ability of this intervention to improve pediatric emergency provider communication as perceived by patients/families and improve provider self-efficacy in pediatric-specific communication skills for multidisciplinary pediatric providers ${ }^{15,16}$ and thus we aimed to determine whether these improvements also translated into an enhanced patient/family experience.

\section{Methods}

\section{Setting and Participants}

The study was conducted at Texas Children's Hospital (TCH) in Houston, Texas, a quaternary care pediatric and women's care hospital, in collaboration with the Academy of Communication in Healthcare (ACH). Providers (physicians and advanced practice providers) from anesthesia, surgery, obstetrics/gynecology, community pediatrics, pediatric hospital medicine, pediatric emergency medicine, and various ambulatory and inpatient pediatric subspecialties were invited to participate in a 5.5-hour RCC skills training course. ACHtrained TCH providers taught over 1000 multidisciplinary providers from July 1, 2016 to July 1, 2019. Forty-nine pediatric hematology/oncology providers took the course during this time period. An Institutional Review Board approval was obtained for the study.

\section{Intervention}

The interactive course was divided into 3 skill sets within a pediatric patient encounter: (1) Beginning the encounter , (2)Relationship-centered interviewing, and (3) Ending the encounter. Participants utilized these 3 skill sets in order to establish rapport, convey empathy, elicit patient/family perspectives, relay information, and make collaborative decisions with the patient/family. Each course had no more than 12 participants and was led by $2 \mathrm{ACH}$-trained TCH providers over 5.5 hours using a combination of didactic learning, small group discussions, and role-play skills practice. ACH-trained TCH providers were a multidisciplinary group 
from various subspecialties, including 4 pediatric subspecialists, 1 pediatric critical care advanced practice provider, 1 pediatric surgery advanced practice provider, 2 pediatric surgeons, 2 pediatric hospitalists, 2 general pediatricians, and 1 pediatric anesthesiologist.

\section{Measures}

Self-reported demographic data was collected via pre-course survey for the 49 pediatric hematology/oncology providers. Patient experience was measured using the overall care provider section score and 5 care provider questions from Press Ganey email surveys (PGS) completed by patients/families: (1) Care provider information about medications, (2) Care provider concern for questions/worries, (3) Care provider efforts to include in decisions , (4) Care provider spoke using clear language, and (5) Likelihood of recommending care provider. PGS were collected for a minimum of 12 months pre-course and at least 3 months post-course. Only providers with both pre-course and post-course PGS submissions were included in the data analysis.

\section{Statistical Analysis}

Provider PGS data was reported as mean score \pm standard deviation and changes over time were analyzed by mixed effect models. Statistical significance was initially defined as $P<0.05$ and was adjusted using a stepdown multiple testing correction correcting for 18 tests for the PGS data. Analyses were carried out in the SAS v 9.4 software (SAS Inc., Cary, NC).

\section{Results}

A total of 49 pediatric hematology/oncology providers took the course, and demographic data is shown in Table 1. The majority of participants were physicians (38 providers, $77.6 \%)$, less than 45 years of age (36 providers, $73.5 \%$ ), female (42 providers, $85.7 \%$ ), in practice for 10 or fewer years (30 providers, $61.2 \%$ ), and cared for patients in both inpatient and outpatient settings (27 providers, $55.1 \%$ ).

Of the 49 participants, 15 had both pre- and post-course PGS completed by patients/families. As shown in Table 2, a total of 98 pre-course and 262 post-course PGS were available for analysis, and providers' mean overall section score and mean score for each of the 5 PGS questions significantly improved from pre-course to post-course $(P<0.05)$. After a stepdown multiple testing correction, the difference in providers' mean scores still demonstrated a strong improvement post-course, but this improvement was no longer considered statistically significant.

\section{Discussion}

This study demonstrates improvement in specific aspects of patient/family experience for pediatric hematology/oncology providers who chose to undergo RCC skills training. Not only were patients/families more likely to recommend the provider to others after the RCC skills training, but patients/families also specifically reported an improved experience with regard to the information shared about medications, the provider's concern for patient's/family's questions and worries, the provider's efforts to include the patient/family in decision-making, and the provider's use of clear language.

This study's RCC skills course offers an auspicious avenue to fulfill the need for communication training within the field of pediatric hematology/oncology, where providers report they do not feel prepared for difficult conversations. ${ }^{8,17-19}$ In 2012, only a third of pediatric hematology/oncology fellows in the United States and Canada reported receiving formal communication training in fellowship. ${ }^{18}$ Similarly, in 2015, only about half of pediatric hematology/oncology fellowship program directors in the United States reported having a formal communication training program. ${ }^{19}$ Furthermore, the most common methods reported in these surveys were lectures or case scenarios, rather than experiential-based learning methods.

In a 2016, a review by Feraco et al. called for a revitalization of communication skills training in pediatric hematology/oncology. The review specifically advocated for moving beyond role modeling and highlighted workshops' effective utilization of the experiential learning cycle through a combination of didactic learning, skills practice and reflective feedback. ${ }^{20}$ Similarly, data within pediatric hematology/oncology and pediatric critical care suggests that workshops are one of the most valuable mechanisms for communication training 
in difficult conversations. ${ }^{21}$ Although there are barriers to implementing these training strategies, such as time, cost, and lack of faculty expertise in communication skills, even small pediatric hematology/oncology programs (defined as less than 4 fellows per year) have demonstrated the feasibility of such an initiative. ${ }^{19}$ Current literature suggests specific adjustments, such as the utilization of role play (instead of standardized patients) and investment in home institution faculty development, as ways to pragmatically reduce long term cost for fellowship and faculty programs without significant sacrifices in quality. ${ }^{18,19}$ Both role play and faculty development were utilized in the current study. Additionally, some pediatric hematology/oncology fellowship programs have recently initiated experiential-based communication skills training with success. ${ }^{18-21}$ These new training initiatives within pediatric hematology/oncology, along with the current study, strengthen the growing foundation of evidence that communication training that focuses on the patient-provider relationship has the power to change both patient satisfaction and patient outcomes. ${ }^{4,12,22-24}$

Additionally, although the current study's intervention was an RCC skills course designed for a multidisciplinary group of pediatric and women's health providers, the course's 3 skill sets explicitly address the NCI's 6 core communication functions in oncology (fostering healing relationships, exchanging information, responding to emotions, managing uncertainty, making decisions, and enabling patient/family self-management ) as well as two novel pediatric functions (providing validation and supporting hope) described by Sisk et al. ${ }^{14}$ This course is also congruent with more recent studies focused on the promotion of specific practices to enhance providers' meaningful connections with patients ${ }^{25}$ and the transformative potential of RCC for medical education and healthcare in the $21^{\text {st }}$ century. ${ }^{26,27}$

The limitations of the current study include a relatively small sample size $(n=49)$ and the lack of availability of endpoint data for all providers $(n=15)$. Although pediatric hematology/oncology fellows have undergone similar communication training at our institution, PGS are not an appropriate outcome to measure the effect on patient experience for fellows, and an expansion of outcome measures is needed to help assess the impact for fellows in the future. Lastly, similar to critique in current literature regarding the impact of communication skills training, this study was not a randomized-controlled trial, ${ }^{28}$ and a multi-institutional, randomized-controlled study is needed for further validation of the effect on patient/family experience.

In conclusion, we have demonstrated that RCC skills training that focuses on establishing rapport, conveying empathy, eliciting patient/family perspectives, relaying information, and making collaborative decisions with the patient/family is a valuable tool to engender effective communication skills in pediatric hematology/oncology providers to improve patient/family experience. We established our RCC skills training through a collaboration with the $\mathrm{ACH}$ and partnered didactics with extensive role-play in a multidisciplinary environment to optimize engagement and experiential learning (Fig. 1). Our experience contributes to increasing evidence that there are a variety of resources and learning environments from which institutions can choose to build a successful program.

\section{Authors' Disclosure of Potential Conflicts of Interest}

The authors declare that there are no conflicts of interest.

\section{Acknowledgement s}

The authors thank Lorianne Classen, MPH, for her oversight in organizing participants and collecting data and Devin Olivares-Reed, BS, for his organization of data for this study. The authors also thank Press Ganey Associates and Academy of Communication in Healthcare (ACH) for their collaboration, and specifically ACH Faculty Calvin Chou, MD, PhD, and Cara Myers, CNM, for their expertise in training our cohort of ACH-trained providers.

\section{References}

1. Pew-Fetzer Task Force on Advancing Psychosocial Health Education.Health Professions Education and Relationship-Centered Care. San Francisco; 1994.

2. Beach MC, Inui T, Frankel R, et al. Relationship-centered care: A constructive reframing. J Gen Intern 
Med . 2006;21(SUPPL. 1):S3-S8. doi:10.1111/j.1525-1497.2006.00302.x

3. Tamblyn R, Abrahamowicz M, Wenghofer E, Blackmore D, Winslade N. Physician Scores on a National Clinical Skills Examination as Predictors of Complaints. JAMA . 2007;298(9):993-1001.

4. Kelley JM, Kraft-Todd G, Schapira L, Kossowsky J, Riess H. The influence of the patient-clinician relationship on healthcare outcomes: A systematic review and meta-analysis of randomized controlled trials. $P L o S$ One . 2014;9(4). doi:10.1371/journal.pone.0094207

5. Street RL. Physicians' communication and parents' evaluations of pediatric consultations. Med Care . 1991;29(11):1146-1152. doi:10.1097/00005650-199111000-00006

6. Levetown M. Communicating with children and families: From everyday interactions to skill in conveying distressing information.Pediatrics . 2008;121(5):e1441-e1460. doi:10.1542/peds.2008-0565

7. Sisk BA, Baker JN. Microethics of Communication - Hidden Roles of Bias and Heuristics in the Words We Choose. JAMA Pediatr . 2018;172(12):1115-1116. doi:10.1002/pon.1223

8. Sisk BA, Mack JW, Ashworth R, DuBois J. Communication in pediatric oncology: State of the field and research agenda. Pediatr Blood Cancer . 2018;65(1):1-10. doi:10.1002/pbc.26727

9. Ranmal R, Prictor M, Jt S, Ranmal R, Prictor M, Jt S. Interventions for improving communication with children and adolescents about their cancer. Cochrane Database Syst Rev . 2008;4:CD002969. doi:10.1002/14651858.CD002969.pub2.www.cochranelibrary.com

10. Hentea C, Cheng ER, Bauer NS, Mueller EL. Parent-centered communication at time of pediatric cancer diagnosis: A systematic review. Pediatr Blood Cancer . 2018;65(8). doi:10.1002/pbc.27070

11. Lin B, Gutman T, Hanson CS, et al. Communication during childhood cancer: Systematic review of patient perspectives. Cancer . 2020;126(4):701-716. doi:10.1002/cncr.32637

12. Mack JW, Kang TI. Care experiences that foster trust between parents and physicians of children with cancer [published online ahead of print, 2020 Aug 22]. Pediatr Blood Cancer . 2020;67:e28399. doi:10.1002/pbc.28399

13. Epstein RM, Street RL. Patient-Centerd Communication in cancer care.J Nat Cancer Inst . 2007;1:1-222. doi:NIH Publication No. 07-6225.

14. Sisk BA, Friedrich A, Blazin LJ, Baker JN, Mack JW, DuBois J. Communication in Pediatric Oncology: A Qualitative Study.Pediatrics . 2020;146(3):e20201193. doi:10.1542/peds.2020-1193

15. Leaming-Van Zandt KJ, Zhu H, Banuelos RC, Lopez MA, Hsu DC. Impact of a Pediatric-Focused Communication Course on Patient/Caregiver-Perceived Physician Communication Skills in a Pediatric Emergency Department. Pediatr Emerg Care . 2019;Publish Ah(00):1-8. doi:10.1097/pec.0000000000001950

16. Grome LJ, Banuelos RC, Lopez MA, Nicome RK, Leaming-Van Zandt KJ. Communication course for pediatric providers improves self-efficacy.Plast Reconstr Surg - Glob Open . 2018;6(10):1-8. doi:10.1097/GOX.0000000000001964

17. Hilden JM, Emanuel EJ, Fairclough DL, et al. Attitudes and practices among pediatric oncologists regarding end-of-life care: Results of the 1998 American Society of Clinical Oncology survey. J Clin Oncol . 2001;19(1):205-212. doi:10.1200/JCO.2001.19.1.205

18. File W, Bylund CL, Kesselheim J, Leonard D, Leavey P. Do pediatric hematology/oncology (PHO) fellows receive communication training? Pediatr Blood Cancer . 2014;61(3):502-506. doi:10.1002/pbc.24742

19. Weintraub L, Figueiredo L, Roth M, Levy A. The feasibility of implementing a communication skills training course in pediatric hematology/oncology fellowship. Pediatr Hematol Oncol . 2016;33(7-8):480-490. doi:10.1080/08880018.2016.1240279 
20. Feraco AM, Brand SR, Mack JW, Kesselheim JC, Block SD, Wolfe J. Communication Skills Training in Pediatric Oncology: Moving Beyond Role Modeling. Pediatr Blood Cancer . 2008;50(November 2015):10181025. doi: $10.1002 / \mathrm{pbc}$

21. Kersun L, Gyi L, Morrison WE. Training in difficult conversations: A national survey of pediatric hematology-oncology and pediatric critical care physicians. J Palliat Med . 2009;12(6):525-530. doi:10.1089/jpm.2008.0251

22. Merckaert I, Delevallez F, Gibon AS, et al. Transfer of communication skills to the workplace: Impact of a 38-hour communication skills training program designed for radiotherapy teams. J Clin Oncol . 2015;33(8):901-909. doi:10.1200/JCO.2014.57.3287

23. Epstein RM, Duberstein PR. Effect of a Patient-Centered Communication Intervention on OncologistPatient Communication, Quality of Life, and Health Care Utilization in Advanced Cancer: The VOICE Randomized Clinical Trial Ronald. JAMA Oncol . 2017;3(1):92-100. doi:10.1016/j.physbeh.2017.03.040

24. Moore PM, Rivera S, Bravo-Soto GA, Olivares C, Lawrie TA. Communication skills training for healthcare professionals working with people who have cancer. Cochrane Database Syst Rev . 2018;(7):CD003751. doi:10.1002/14651858.CD003751.pub4.www.cochranelibrary.com

25. Zulman DM, Haverfield MC, Shaw JG, et al. Practices to Foster Physician Presence and Connection with Patients in the Clinical Encounter. JAMA . 2020;323(1):70-81. doi:10.1001/jama.2019.19003

26. Weiss T, Swede MJ. Transforming Preprofessional Health Education Through Relationship-Centered Care and Narrative Medicine. Teach Learn Med . 2019;31(2):222-233. doi:10.1080/10401334.2016.1159566

27. Wyer PC, Alves Silva S, Post SG, Quinlan P. Relationship-centred care: antidote, guidepost or blind alley? the epistemology of 21st century health care. J Eval Clin Pract . 2014;20(6):881-889. doi:10.1111/jep.12224

28. Alelwani SM, Ahmed YA. Medical training for communication of bad news: A literature review. $J$ Educ Health Promot . 2014;3(51). doi:10.4103/2277-9531.134737

\section{Legend}

FIGURE 1 Process model for developing a relationship-centered communication skills training workshop. A thorough resource assessment identifies strengths and capacities that are invaluable in the development process. Common barriers such as cost, time, accessibility, and lack of expertise have multiple potential solutions. ${ }^{19}$ A comprehensive, multidisciplinary workshop including didactics, hands-on skills practice through role playing, and reflective feedback engages the experiential learning cycle for optimal learning. ${ }^{18-20}$ Optimal skill acquisition then leads to relationship-centered care and transformational change for both the patient/family and provider. ${ }^{1}$

\section{Hosted file}

BTC PHO Manuscript Tables_FINAL.pdf available at https://authorea.com/users/366767/articles/ 486411-relationship-centered-communication-training-for-pediatric-hematology-oncologyproviders-to-improve-patient-experience 


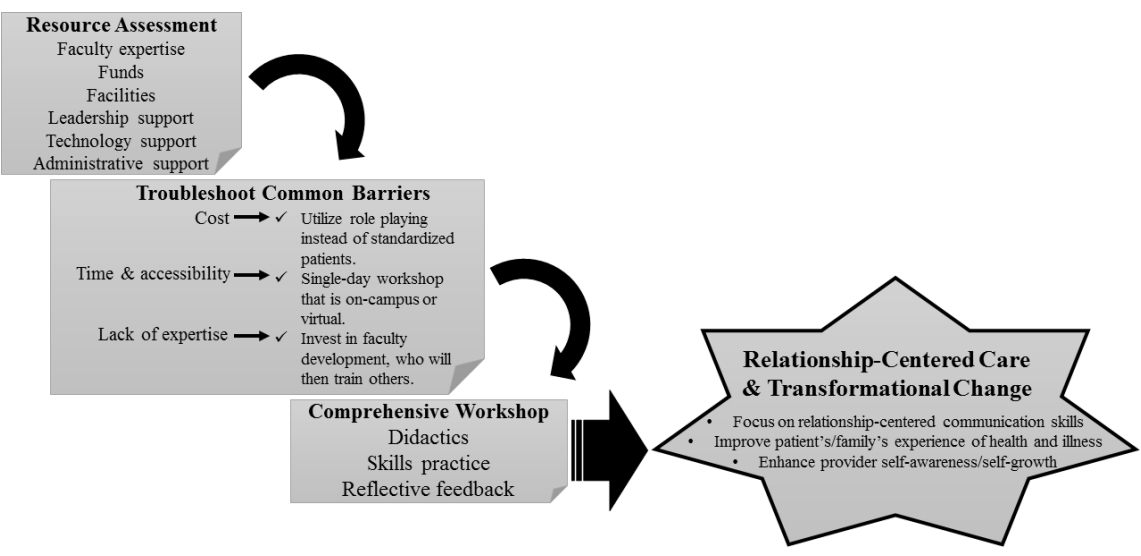

